\title{
Germinação de Sementes de Anadenanthera peregrina (L.) Speg. com Diferentes Substratos em Condições Laboratoriais
}

\author{
Cristiana do Couto Miranda ${ }^{1,2}$, Denivan Melo dos Santos Souza ${ }^{1,2}$, \\ Pedro Ramon Manhone ${ }^{1,2}$, Paulo Cezar de Oliveira ${ }^{1}$, Tiago Böer Breier ${ }^{1}$ \\ ${ }^{1}$ Instituto de Florestas, Universidade Federal Rural do Rio de Janeiro - UFRRJ \\ ${ }^{2}$ Programa de Pós-Graduação em Ciências Ambientais e Florestais, \\ Universidade Federal Rural do Rio de Janeiro - UFRRJ
}

\section{RESUMO}

Em sementes de espécies florestais, geralmente ocorre grande variação no desempenho germinativo em relação aos substratos em laboratório, sendo necessário determinar o tipo de substrato que proporciona a melhor germinação para cada espécie florestal. Este estudo objetivou avaliar o comportamento de germinação (porcentagem de germinação e índice de velocidade de germinação - IVG) das sementes da leguminosa florestal Anadenanthera peregrina (L.) Speg. (Fabaceae - Mimosoideae), submetidas a diferentes substratos (areia, papel filtro e vermiculita). Os substratos que demonstraram maiores valores de IVG foram vermiculita e papel filtro, sendo que a vermiculita também propiciou maior porcentagem de germinação, indicando que esse substrato é o mais adequado para testes de germinação de sementes de A. peregrina.

Palavras-chave: espécie florestal, Anadenanthera peregrina, germinação, substrato de germinação.

\section{Seed Germination of Anadenanthera peregrina (L.) Speg. with Different Substrates under Laboratory Conditions}

\begin{abstract}
In seeds of forest species, usually there is a great variation in the seed germination performance in relation to the substrate in laboratory, so it is necessary to determine the type of substrate which allows a better germination for each forest species. This study aimed at evaluating the behavior of seed germination (percentage of germination and index of germination speed - IVG) of the legume forest species Anadenanthera peregrina (L.) Speg. (Fabaceae - Mimosoideae), submitted to different substrates (sand, filter paper and vermiculite). The substrates that demonstrated higher values of IVG were vermiculite and filter paper, and vermiculite also allowed higher percentage of germination, indicating that this substrate is the most adequate for germination tests of seeds of A. peregrina.
\end{abstract}

Keywords: forest species, Anadenanthera peregrina, seed germination, substrates to germination. 


\section{INTRODUÇÃO}

Anadenanthera peregrina (L.) Speg., membro da família Fabaceae, subfamília Mimosoideae, é popularmente conhecida como angico-domorro, angico-branco, angico-vermelho, angico, paricá-de-curtume, paricá e paricá-da-terra-firme (Lorenzi, 2009). Segundo o mesmo, essa espécie possui ampla distribuição geográfica, ocorrendo do Estado de Tocantins ao Estado do Rio de Janeiro, em Floresta Estacional Semidecidual e na sua transição para o Cerrado. É uma espécie pioneira, que ocorre, especialmente, em formações primárias e secundárias, tanto em solos pedregosos e arenosos como em argilosos, desde que sejam bem drenados.

Entre os principais usos, destaca-se, na A. peregrina, seu potencial apícola, medicinal; também, por causa da elevada densidade da sua madeira $\left(1,08 \mathrm{~g} . \mathrm{cm}^{-3}\right)$, pode ser utilizada na construção civil, na confecção de móveis e esquadrias, bem como para lenha e carvão (Lorenzi, 2009). Além disso, essa espécie é recomendada para recuperação de áreas degradadas, servindo de poleiros para aves e também como fonte de diásporos, de forma a contribuir para a chuva de sementes no local (Araújo et al., 2006). No entanto, ainda são poucos os estudos referentes à sua propagação em laboratório.

De forma geral, a ampla utilização das espécies tropicais nativas em práticas conservacionistas ou de recuperação de áreas degradadas não tem acontecido em virtude da ausência de conhecimento consolidado sobre a biologia e a ecologia, bem como sobre as técnicas de propagação e manejo dessas espécies (Carpi et al., 1996; Ranieri et al., 2003). As sementes são a via de propagação mais empregada na implantação de plantios e a busca de conhecimentos sobre as condições ótimas para a germinação é fundamental dentro da pesquisa, além de fornecer informações valiosas sobre a propagação das espécies (Varela et al., 2005). Os estudos sobre a germinação de sementes são essenciais para se proporem técnicas eficientes para a exploração das espécies nativas (Perez et al., 2001).

Os testes de germinação realizados em laboratório - sob condições ideais de temperatura, teores de umidade e substrato - objetivam avaliar o máximo potencial de germinação do lote de sementes
(Figliolia et al., 1993). As Regras para Análise de Sementes (RAS) (Brasil, 2009) recomendam alguns substratos, como papel toalha, filtro ou mata-borrão, areia e solo. No entanto, existem poucas recomendações para as espécies florestais (Souza et al., 2007) e outros substratos vêm sendo testados, como é o caso da vermiculita.

Segundo Abreu et al. (2005), mesmo que os embriões estejam completamente maduros, o substrato é um fator importante tanto para a velocidade quanto para porcentagem de germinação. De acordo com Figliolia et al. (1993) e Novembre (1994), a utilização do substrato adequado é fundamental, pois este tem função de suprir as sementes de água, além de proporcionar condições para a germinação das mesmas e o desenvolvimento das plântulas.

A velocidade de desenvolvimento é um dos parâmetros mais utilizados para determinação do vigor de sementes, sendo usado, principalmente, o índice de velocidade de germinação. Esse índice baseia-se no pressuposto de que sementes mais vigorosas germinarão mais rapidamente (PiñaRodrigues et al., 2004).

A partir do teste de germinação, é possível conhecer o substrato que potencializa o processo de germinação de A. peregrina, proporcionando a expressão máxima do vigor; nesse intuito, esta se constitui numa etapa essencial para definir um método adequado para avaliação da qualidade das sementes. Nesse foco, o presente estudo objetivou avaliar o comportamento germinativo, por meio das porcentagens de germinação e do índice de velocidade de germinação das sementes de A. peregrina (angicodo-morro), submetidas a diferentes substratos (areia, papel filtro e vermiculita) em condições de laboratório.

\section{MATERIAL E MÉTODOS}

As sementes de Anadenanthera peregrina (L.) Speg. foram coletadas de duas árvores matrizes na Fazenda Cachoeirão, município de Além-ParaíbaMG, em setembro de 2009. O material botânico foi identificado com ajuda de um botânico e incorporado ao herbário do Departamento de Botânica da UFRRJ. Os frutos foram secos ao ar 
livre, sob luz solar, durante o período de dois dias. Esse procedimento facilitou o beneficiamento das sementes. A redução do teor de água das sementes não compromete sua viabilidade, pois as mesmas são ortodoxas. As sementes foram armazenadas durante duas semanas em câmara fria a $10^{\circ} \mathrm{C}$, até o início do teste.

Os diferentes substratos utilizados para avaliar o comportamento germinativo das sementes de A. peregrina foram areia, papel filtro e vermiculita. Foram utilizadas dez repetições por tratamento, sendo 20 sementes para cada repetição, totalizando 600 sementes $(35,68 \mathrm{~g})$. Foram utilizadas caixas "gerbox" preenchidas com o substrato referente ao tratamento, sendo admitido o mesmo volume de substrato para os tratamentos areia e vermiculita $\left(135 \mathrm{~cm}^{3}\right)$. No tratamento com papel filtro, foram utilizadas duas folhas desse papel. Os materiais usados no experimento foram todos esterilizados em autoclave, com exceção das gerbox plásticas. A semeadura foi feita sobre os substratos em todos os tratamentos e, posteriormente, estes foram umedecidos com água, sendo utilizados os seguintes volumes iniciais: $40 \mathrm{~mL}$ no tratamento com areia, $15 \mathrm{~mL}$ no papel filtro e $60 \mathrm{~mL}$ no tratamento com vermiculita. A diferença de volumes deveu-se às distintas capacidades de retenção de água. Os "gerbox" com as sementes foram colocados em uma câmara germinadora a $30^{\circ} \mathrm{C}$, com luz constante. A água evaporada foi reposta diariamente, de acordo com a necessidade de cada substrato.

Os experimentos de germinação das sementes foram realizados nos germinadores tipo Biochemical Oxigen Demand (B.O.D.), do Laboratório de Biologia Reprodutiva, Sementes e Conservação de Espécies Arbóreas (LACON) - Departamento de Silvicultura no Instituto de Florestas da Universidade Federal Rural do Rio de Janeiro.

O número de sementes germinadas foi avaliado diariamente, adotando-se o critério botânico, segundo Castellani et al. (2009), que considera as sementes germinadas após a emissão da radícula. Foram avaliados os parâmetros porcentagem de germinação e índice de velocidade de germinação (IVG). A germinação foi avaliada a partir da porcentagem de sementes germinadas até o sexto dia após a semeadura. $\mathrm{O}$ teste foi encerrado quando se observou que as sementes não germinavam mais, sendo as mesmas examinadas e então verificado que o tecido estava em estado de deterioração.

Para cada tratamento, foi calculado o IVG sugerido por Biruel et al. (2007), o qual evidencia o número de sementes germinadas por dia sendo o IVG $=\mathrm{G} 1 / \mathrm{N} 1+\mathrm{G} 2 / \mathrm{N} 2+\ldots \mathrm{Gn} / \mathrm{Nn}$, em que G1, G2... Gn é igual ao número de sementes germinadas, e N1, N2... Nn corresponde ao número de dias. Os dados foram submetidos a testes de normalidade (Teste de Lilliefors) e homogeneidade (Teste de Bartlett), e então comparados por meio do Teste de Tukey a $5 \%$ de probabilidade, quando a análise de variância realizada foi significativa (Zar, 1999). Não houve necessidade de transformações dos dados, pois os mesmos apresentaram normalidade e homogeneidade de variâncias.

\section{RESULTADOS E DISCUSSÃO}

O substrato que proporcionou maior porcentagem de germinação das sementes de Anadenanthera peregrina, no sexto dia após semeadura (último dia do experimento), foi a vermiculita (Tabela 1), diferindo significativamente dos demais (ANOVA, $\mathrm{F}=9,7 ; P=0,0007 ; \mathrm{n}=30$ ).

As sementes de A. peregrina iniciaram a emissão da radícula no segundo dia após serem semeadas nos substratos, demonstrando, de forma geral, elevada velocidade de germinação dessa espécie (Figura 1).

Tabela 1. Valores médios de germinação (\%) e do índice de velocidade de germinação (IVG) de sementes de Anadenanthera peregrina em diferentes substratos em condições laboratoriais.

Table 1. Seed germination (\%) and germination speed index (IVG) of Anadenanthera peregrina seeds in different substrates at laboratory.

\begin{tabular}{lcc}
\multicolumn{1}{c}{ Substrato } & Germinação (\%) & IVG \\
\hline Areia & $77,0( \pm 13,9) \mathrm{B}$ & $14,3( \pm 2,1) \mathrm{B}$ \\
Papel filtro & $85,5( \pm 5,9) \mathrm{B}$ & $18,7( \pm 1,4) \mathrm{A}$ \\
Vermiculita & $96,0( \pm 3,9) \mathrm{A}$ & $17,8( \pm 3,1) \mathrm{A}$ \\
CV $(\%)$ & 5,5 & 13,9 \\
\hline
\end{tabular}

Médias de dez repetições. Valores seguidos pela mesma letra na coluna não diferem entre si pelo Teste Tukey $(\mathrm{p}<0,05)$. Valores entre parênteses correspondem ao desvio padrão. $\mathrm{CV}=$ coeficiente de variação. 


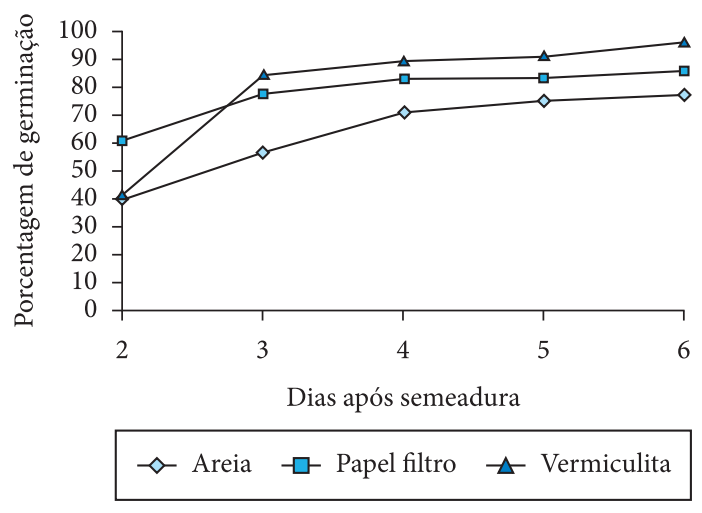

Figura 1. Porcentagem acumulada de germinação de sementes de Anadenanthera peregrina em diferentes substratos, em condições laboratoriais.

Figure 1. Cumulative germination percentage of seeds of Anadenanthera peregrina in different substrates at laboratory.

Dentre os substratos analisados, foi verificado que a germinação é mais lenta quando as sementes de $A$. peregrina são colocadas em areia (ANOVA, $\mathrm{F}=9,5 ; P=0,001 ; \mathrm{n}=30)$. Para a vermiculita e o papel filtro, os índices de velocidades de germinação (IVG) não diferiram significativamente, sendo esses os substratos que apresentaram maior velocidade de germinação (Tabela 1). Embora tenha ocorrido maior porcentagem de sementes germinadas inicialmente no papel filtro, observa-se que houve um maior incremento na porcentagem de germinação na vermiculita, a partir do terceiro dia após semeadura, o que pode ter compensado o IVG (Figura 1).

A capacidade de retenção de água apresentada por cada substrato aliada às características das sementes, que regulam o fluxo de água para seu interior, pode ter influenciado na porcentagem de germinação.

De acordo com Figliolia et al. (1993), a areia é um substrato que possui alta capacidade de drenagem, fazendo com que a água passe rapidamente para as camadas subsuperficiais, o que deixa a parte superior desse substrato ressecada. Dessa forma, o processo de embebição de água das sementes pode ser diminuído. Essa capacidade de drenagem é em decorrência da estrutura física da areia, que apresenta alta porosidade e partículas de maior diâmetro, tendo menor eficiência na absorção de moléculas de água por causa da sua menor área superficial (Reis et al., 2002).
Scalon et al. (2003) também observaram que o substrato de areia não é o mais adequado para a germinação de Caesalpinia pelthophoroides Benth. Diferentemente, Coelho et al. (2006) não encontraram diferenças significativas ao avaliarem germinação de sementes de Schizolobium parahyba (Vell.) Blake em areia, vermiculita e esterco mais terra vegetal. De acordo com Bocchese et al. (2008), essas contradições observadas na literatura demonstram que as sementes de espécies florestais possuem diferentes necessidades de substrato, o que evidencia a necessidade de testes para o conhecimento dos melhores substratos a serem utilizados para as diferentes espécies.

O substrato de papel, por sua vez, permite facilmente a evaporação da água, mesmo fazendo-se reposição periódica de água (Machado et al., 2002), como foi feito no presente estudo. Esse fato pode ter contribuído para a menor porcentagem de germinação em relação à vermiculita, pois, segundo Marcos Filho et al. (1987), a uniformidade da umidade do substrato durante o teste de germinação é um fator primordial.

Por outro lado, o favorecimento à germinação de sementes proporcionado pela vermiculita também foi verificado por Pereira \& Andrade (1994); Vidigal et al. (2007) atribuíram tal germinação ao fato de esse substrato possuir alta capacidade de retenção de água e proporcionar condições adequadas de aeração em função das suas propriedades físicoquímicas. Essas características são fundamentais ao substrato, de forma a evitar a anaerobiose, que, segundo Silva \& Carvalho (2008), reduz a porcentagem e a velocidade de germinação.

As sementes de A. peregrina apresentam características morfológicas e ecológicas que contribuem para ampla dispersão anemocórica. O fruto legume achatado e deiscente comporta sementes achatadas dispersadas pelo vento (Costa et al., 2003). Segundo Carvalho et al. (2006) e Pinho et al. (2009), pelo fato de essas sementes serem ortodoxas, após a dispersão, elas podem ficar armazenadas no banco de sementes, sendo essa sua estratégia de regeneração. Quando encontram condições favoráveis, tais como presença de umidade e temperatura adequadas, iniciam seu processo de embebição e, por conseguinte, a germinação. Nos 
ambientes naturais, a umidade do solo e as condições microclimáticas são determinantes para o sucesso da germinação das sementes e o estabelecimento dos indivíduos de $A$. peregrina. Em laboratório, essas condições são proporcionadas pelo substrato e condições controladas de temperatura, umidade e luminosidade. Dessa forma, o substrato que melhor favoreça retenção de umidade e condições físicas - como aeração para as sementes, possibilitando o maior desempenho da porcentagem e velocidade de germinação - será mais indicado para os testes germinativos da espécie. Nesse contexto, a vermiculita se destacou por condicionar o maior desempenho na germinação de A. peregrina, sendo indicada para testes de germinação dessa espécie. De acordo com Souza et al. (2007), há grande variação do desempenho germinativo das sementes em relação aos substratos utilizados em condições de laboratório, tornando necessária a definição do ambiente que melhor proporcione a expressão máxima do vigor de cada espécie florestal.

\section{CONCLUSÃO}

Os substratos que demonstraram maiores IVG foram vermiculita e papel filtro, sendo que a vermiculita também propiciou maior porcentagem de germinação, indicando que esse substrato é o mais adequado para a avaliação da qualidade fisiológica de sementes de Anadenanthera peregrina.

\section{STATUS DA SUBMISSÃO}

Recebido: 22/12/2010

Aceito: 15/12/2011

Resumo publicado online: 20/12/2012

Artigo completo publicado: 31/03/2012

\section{AUTOR(ES) PARA CORRESPONDÊNCIA}

\section{Cristiana do Couto Miranda}

Programa de Pós-Graduação em Ciências

Ambientais e Florestais, Universidade Federal Rural do Rio de Janeiro UFRRJ

BR 465, Km 7, CEP 23890-000,

Seropédica, RJ, Brasil

e-mail: coutomiranda@yahoo.com.br

\section{REFERÊNCIAS}

Abreu D, Nogueira AC, Medeiros ACS. Efeito do substrato e da temperatura na germinação de sementes de cataia (Drimys brasiliensis Miers. Winteraceae). Revista Brasileira de Sementes 2005; 27(1):149-157. http://dx.doi.org/10.1590/S0101-31222005000100019

Araújo FS, Martins SV, Meira Neto JAA, Lanis JLL, Pires IE. Estrutura da vegetação arbustivoarbórea colonizadora de uma área degradada por mineração de caulim, Brás Pires, MG. Revista Árvore 2006; 30(1):107-116.

Biruel RP, Aguiar IB, Paula RC. Germinação de sementes de pau-ferro submetidas a diferentes condições de armazenamento, escarificação química, temperatura e luz. Revista Brasileira de Sementes 2007; 29(3):151-159. http://dx.doi.org/10.1590/S0101-31222007000300018

Bocchese RA, Oliveira AKM, Melloto AM, Fernandes V, Laura VA. Efeito de diferentes tipos de solos na germinação de sementes de Tabebuia heptaphylla, em casa telada. Cerne 2008; 14(1):62-67.

Brasil. Ministério da Agricultura Pecuária e Abastecimento. Regras para análise de sementes. Brasília: MAPA/ACS; 2009. 395p.

CarpiSMF,BarbedoCJ,MarcosFilhoJ.Condicionamento osmótico de sementes de Cedrela fissilis Vell. Revista Brasileira de Sementes 1996; 18(2):271-275.

Carvalho LR, Silva EAA, Davide AC. Classificação de sementes florestais quanto ao comportamento no armazenamento. Revista Brasileira de Sementes 2006; 28(2):15-25. http://dx.doi.org/10.1590/ S0101-31222006000200003

Castellani ED, Aguiar IB, Paula RC. Base para padronização do teste de germinação de sementes de espécies arbóreas de Solanum L. Revista Brasileira de Sementes 2009; 31(2):77-85. http://dx.doi.org/10.1590/ S0101-31222009000200009

Coelho RRP, Silva MTC, Bruno RLA, Santana JSA. Influência de substratos na formação de mudas de guapuruvu (Schizolobium parayba (Vell.) Blake). Revista Ciência Agronômica 2006; 37(2):149-152.

Costa RB, Contini AZ, Melo ESP. Sistema reprodutivo de Anadenanthera peregrina (L.) Speg e Vochysia haenkiana (Spreng.) Mart. em fragmento de cerrado na Chapada dos Guimarães - MT. Ciência Rural 2003; 33(2).

Figliolia MB, Oliveira EC, Piña-Rodrigues FCM. Análise de sementes. In: Aguiar IB, Piña-Rodrigues FCM, Figliolia MB, coordenadores. Sementes florestais tropicais. Brasília: ABRATES; 1993. p.137-174.

Lorenzi H. Árvores Brasileiras: Manual de identificação e cultivo de plantas arbóreas nativas do Brasil. 3rd ed. São Paulo: Instituto Plantarum, 2009. 384 p. v.2. 
Machado CF, Oliveira JA, Davide AC, Guimarães RM. Metodologia para condução do teste de germinação em sementes de Ipê amarelo (Tabebuia serratifolia (Vahl) G. Nicholson). Cerne 2002; 8(2):17-25.

Marcos Filho J, Cícero SM, Silva WR. Avaliação da qualidade das sementes. Piracicaba: FEALQ, 1987.230 p.

Novembre ADLC. Estudo da metodologia para a condução do teste de germinação em sementes de algodão (Gossypium hirsutum L.) deslintadas mecanicamente [tese]. Piracicaba: Escola superior de Agricultura Luiz de Queiroz; 1994.

Pereira TS, Andrade ACS. Germinação de Psidium guajara L. e Passiflora edulis S. - efeito da temperatura, do substrato e morfologia do desenvolvimento pós-seminal. Revista Brasileira de Sementes 1994; 16(1):58-62.

Perez SCJGA, Fanti SC, Casali CA. Influência da luz na germinação de sementes de canafístula submetidas ao estresse hídrico. Bragantia 2001; 60(3):155-156. http://dx.doi.org/10.1590/S0006-87052001000300002

Piña-Rodrigues FCM, Figliolia MB, Peixoto MC. Testes de qualidade. In: Ferreira AG, Borghetti F, organizadores. Germinação: do básico ao aplicado. Porto Alegre: Artmed; 2004. p. 283-297.

Pinho DS, Borges EEL,Corte VB, Nasser BLC. Avaliação da qualidade fisiológica de sementes de Anadenanthera peregrina (L.) Speg. durante o armazenamento. Revista Árvore 2009; 33(1):27-33. http://dx.doi.org/10.1590/ S0100-67622009000100004

Ranieri BD, Lana TC, Negreiros D, Araújo LM, Fernandes GW. Germinação de sementes de Lavoisiera cordata Cogn. e Lavoisiera francavillana Cogn. (Melastomataceae), espécies simpátricas da Serra do Cipó, Brasil. Acta Botanica Brasílica 2003; 17(4):523-530. http://dx.doi.org/10.1590/S0102-33062003000400005
Reis EF, Schaefer CEGR, Vieira LB, Souza CM, Fesnandez HC. Avaliação de contato semente-solo em um solo argiloso sob plantio direto, com diferentes teores de água no solo. Engenharia na Agricultura 2002; 10(1-4):205-237.

Scalon SPQ, Mussury RM, Almeida KA, Rigoni MR. Efeito do álcool e substrato na germinação de sementes de sibipiruna (Caesalpinia pelthophoroides Benth.) colhidas no chão e retiradas da vagem. Ciência e Agrotecnologia 2003; 27(2):389-392. http://dx.doi. org/10.1590/S1413-70542003000200019

Silva BMS, Carvalho NM. Efeito do estresse hídrico sobre o desempenho germinativo da semente de faveira (Clitoria fairchildiana R. A. Howard. Fabaceae) de diferentes tamanhos. Revista Brasileira de Sementes 2008; 30(1):55-65. http://dx.doi.org/10.1590/ S0101-31222008000100008

Souza EB, Pacheco MV, Matos VP, Luiz R, Ferreira C. Germinação de Sementes de Adenanthera Pavonina L. em função de diferentes temperaturas e substratos. Revista Árvore 2007; 31(3):437-443.

Varela VP, Costa SS, Ramos MBP. Influência da temperatura e do substrato na germinação de sementes de itaubarana (Acosmium nitens (Vogel) Yakovlev) - Leguminosae, Caesalpinoideae. Acta Amazonica 2005; 35(1):35-39. http://dx.doi. org/10.1590/S0044-59672005000100006

Vidigal DS, Brasileiro BG, Dias DCFS, Alvarenga EM, Bhering MC. Germinação e morfologia do desenvolvimento pós-seminal de sementes de Nim-indiando (Azadirachta indica A. Juss. - Meliaceae. Revista Brasileira de Sementes 2007; 29(3):35-41. http://dx.doi.org/10.1590/S0101-31222007000300005

Zar JH. Biostatistical analysis. 4rd ed. New Jersey: Prentice-Hall, Inc.; 1999. 663 p. 